\title{
Transient phenomenological modeling of photoelectrochemical cells for water splitting - Application to undoped hematite electrodes
}

\author{
Luísa Andrade, Tânia Lopes, Helena Aguilar Ribeiro, Adélio Mendes* \\ LEPAE - Departamento de Engenharia Química, Faculdade de Engenharia, Universidade do Porto, Rua Dr. Roberto Frias, \\ $s / n$ 4200-465 Porto, Portugal
}

\section{A R T I C L E I N F O}

Article history:

Received 15 July 2010

Received in revised form

24 September 2010

Accepted 28 September 2010

Available online 28 October 2010

Keywords:

Photoelectrochemistry

Modeling

Water splitting

Hydrogen

\begin{abstract}
A B S T R A C T
A phenomenological model is proposed for a better understanding of the basic mechanisms of photoelectrochemical (PEC) cells. The main assumptions of the one-dimensional transient phenomenological model are: i) bulk recombination of the conduction band electrons with holes in the valence band; ii) the mobile charge transport takes place via diffusion, which arises from the concentration profiles, and migration, caused by a macroscopic electric field; iii) negligible effects of microscopic electric fields in the cell and screening effects, as well as negligible Helmholtz and diffuse layers. For modeling purposes, the photoanode was assumed to be a homogeneous nanocrystalline hematite structure, with thickness L, porosity $\varepsilon_{p}$ and tortuosity $\tau$. The TCO/semiconductor interface was modeled as an ideal ohmic contact, while the electrolyte/platinized TCO interface was described by a Butler-Volmer approach. An alkaline electrolyte solution was used, allowing the transport of the ionic species from the counter-electrode to the photoanode. The continuity and transport governing equations are defined for the mobile species involved: electrons in the conduction band of the semiconductor, holes in the valence band and hydroxyl ions in the electrolyte. Simulated I-V characteristics were computed and the corresponding results compared with the experimental values. The simulated results were in straight agreement with the experimental data.
\end{abstract}

(c) 2010 Professor T. Nejat Veziroglu. Published by Elsevier Ltd. All rights reserved.

\section{Introduction}

It is known that world demand for energy is forecasted to duplicate by 2050 and it is also widely recognized that the existing energy networks are not adequate to supply this demand in a sustainable way [1]. The increasing public awareness concerning the carbon dioxide emissions and the enhanced depletion of fossil fuels motivates the development of alternative technologies based on non-polluting and clean energy sources [2,3]. So far, the sun is our primary source of clean and abundant energy, striking $120 \mathrm{PW}$ of radiation onto the surface of earth at any given moment and is the most powerful resource able to cope with the world's energy needs [1]. However, the present technologies that take advantage of solar energy have a limited ability to store energy due to the variability of daily solar radiation and so an effective method to store energy for later dispatch is therefore needed [4,5]. A practical way to overcome this problem is using sunlight to split water into hydrogen and oxygen - photoelectrolysis [3] by means of a photoelectrochemical (PEC) cell. The water split phenomenon was first reported by Fujishima and Honda in 1972, but it was only recently that this technology received

\footnotetext{
* Corresponding author. Tel.: +351 225081695; fax: +351 225081449.

E-mail address: mendes@fe.up.pt (A. Mendes).
}

0360-3199/\$ - see front matter ( 2010 Professor T. Nejat Veziroglu. Published by Elsevier Ltd. All rights reserved. doi:10.1016/j.ijhydene.2010.09.098 
special attention from researchers as a potential renewable energy source [3,6-9].

A photoelectrochemical cell is made of a semiconductor, such as iron oxide $\left(\mathrm{Fe}_{2} \mathrm{O}_{3}\right)$ - hematite - and a counter-electrode (cathode), both immersed in an aqueous electrolyte solution. When exposed to sunlight, the semiconductor absorbs photons with sufficient energy to inject electrons from the valence band to its conduction band, creating electron-hole pairs. The holes exhibit oxidation potential while the electrons are transferred over the external circuit to the cathode to promote water reduction into hydrogen gas. The energy conversion efficiency of such process is therefore highly determined by the structure and properties of the semiconductor and the electronic junction formed at the semiconductor/electrolyte interface $[10,11]$. Presently, these devices achieve very low conversion efficiencies since most of the semiconductors used for this application have a large energy bandgap. Furthermore, the band edge potentials of many semiconductors are not suitable for oxygen and hydrogen evolution and the water split reaction can not take place. This is the case of hematite, whose conduction band edge is positioned at a more positive potential than the reduction potential of water. As a result, an additional bias voltage is required to promote the reduction reaction at the cathode side [3].

In the last decade, several studies focusing on promising photoelectrodes were carried out. Doped and undoped semiconductor materials were synthesized and characterized regarding their photoelectrochemical, structural, electrical and optical properties [8,12-14]. Recent studies report hematite as a promising material with several advantages: it has a suitable bandgap energy around $2.0 \mathrm{eV}$, is able to use $40 \%$ of the incident solar spectrum, it is chemically and thermally stable in aqueous solutions and also toward photocorrosion, its valence band is appropriate for oxygen evolution and it is abundant and a low-cost material $[3,8,15]$. Despite several efforts have been made to develop new more adequate materials for water splitting in photoelectrochemical cells, very few research works focused on the insightful understanding of the working mechanisms of PEC cells. An important work was developed by Gerischer $[16,17]$ on the kinetics and energetics of electron transfer across semiconductor/electrolyte junctions and on the nature of the space-charge layer - the semiconductor layer adjacent to the semiconductor/electrolyte interface. Nozik [18] evaluated the energy level scheme for $\mathrm{p}-\mathrm{n}$ photoelectrolysis cells in order to demonstrate an enhanced efficiency by simultaneously illuminating the $\mathrm{n}$ - and p-type electrodes. Khan and Bockris [19] derived an analytical expression for the resulting photocurrent as a function of the physical properties of an illuminated $\mathrm{p}$-type semiconductor electrode and the neighboring electrolyte solution. Boudjemaa and co-authors [13] studied the transport properties of hematite and its application for hydrogen production. Nevertheless, a global and integrated transient phenomenological model for a better understanding of the phenomena occurring in a PEC cell and how they contribute for its performance has never been presented. Simple models were already developed for other water splitting systems, pointing out some relevant aspects. Choi et al. [20] studied a water electrolysis system with a solid polymer electrolyte by means of a simple model based on the Butler-Volmer kinetics. Also based on the Butler-Volmer approach, Nie et al. [21] modeled a proton exchange membrane water electrolyzer and Marangio et al. [22] studied a high pressure polymer electrolyte membrane water electrolyzer. Ni [23-25] developed an interesting work based on the mathematical modeling of solid oxide steam electrolyzers for hydrogen production.

In the present work, an integrated transient phenomenological model for PEC cells is presented. This model aims at contributing to a better understanding of the phenomena occurring in a PEC cell and how they contribute for its performance. The simulated $I-V$ characteristic curves of the PEC cell allowed the calculation of the cells' performance parameters.

\section{Development of the PEC cell model}

Photoelectrolysis of water is the dissociation of water molecules into hydrogen and oxygen by the direct use of light: the conversion of light into electrical current allows the transformation of a chemical entity - water - into another hydrogen - known to be a preferential energy vector [26]. The principle of operation of a photoelectrochemical cell for water splitting is governed by the kinetics of the charge carriers and reaction mechanisms that take place at the electrodes of the PEC cell. Therefore, the understanding of the electronic processes occurring at the semiconductor nanoparticles level, as well as the dynamics of charge separation/transport and reactive mechanisms in the different interfaces is of great importance. Photons with energy $h \cdot v$ higher than the semiconductor's bandgap generate electron-hole pairs $\left(e_{\mathrm{CB}}^{-}+h_{\mathrm{VB}}^{+}\right)$ in the conduction and valence bands, respectively:

$h v+\mathrm{SC} \rightarrow \mathrm{SC}\left(e_{\mathrm{CB}}^{-}+h_{\mathrm{VB}}^{+}\right)$

In an alkaline media, holes oxidize water to oxygen in the semiconductor surface (photoanode),

$2 \mathrm{H}^{+}+2 \mathrm{OH}^{-} \rightarrow \mathrm{H}_{2} \mathrm{O}+1 / 2 \mathrm{O}_{2}$

while the photoexcited conduction band electrons, transported to the counter-electrode (cathode) of the PEC cell, reduce water to form hydrogen gas:

$2 \mathrm{H}_{2} \mathrm{O}+2 \mathrm{e}^{-} \rightarrow 2 \mathrm{OH}^{-}+\mathrm{H}_{2}$

The mechanisms behind oxidation of water by photogenerated holes are not completely understood and depend most probably on the interfacial properties of the semiconductor. On the other hand, the reduction of water into hydrogen is a kinetically simple process compared to the water oxidation reaction [27]. In fact, the efficiency of the oxidation process occurring at the semiconductor can be limited by several competing electron loss pathways: bulk recombination via bandgap states or direct electron loss to holes in the valence band; surface recombination; electron loss from the conduction band; photocorrosion of the semiconductor; and dissolution reactions. The last two processes are responsible for the degradation of the electrode and for the consequent stability problems [28]. 
Fig. 1 shows a scheme of the modeled PEC cell. The photoanode consists of a homogeneous nanocrystalline hematite structure, with thickness $L$, porosity $\varepsilon_{p}$ and tortuosity $\tau$. The photoanode is deposited onto a transparent conductive substrate (TCO), defining the interface TCO/semiconductor at $x=0$. This interface is modeled as an ideal ohmic contact. Since the semiconductor considered herein is a hematite film, an alkaline electrolyte solution should be used, such as sodium hydroxide, allowing the transport of the ionic species from the counter-electrode, a platinum wire, to the photoanode [6]. At $x=b$, the electron transfer phenomenon occurring at the electrolyte/platinized TCO interface is described by the Butler-Volmer equation. The semiconductor/electrolyte interface is defined at $x=L$. The mobile charges considered in the development of the present model are the electrons in the conduction band of the semiconductor, $e^{-}$, the holes in the valence band, $h^{+}$, and the hydroxyl ions in the electrolyte.

For simplicity, the model considers a cell design where the photoelectrode faces its counter-electrode and the space in between is filled with the electrolyte solution. However, typical current-voltage characterization of the PEC cells are performed in a cell configuration known as "cappuccino" [29], in which the surface of the platinum counter-electrode is far away from the semiconductor/electrolyte interface. Hence, in the present model $b>>$ L. Moreover, the cell is irradiated perpendicularly to its photoelectrode and it is assumed that each absorbed photon injects one electron into the hematite conduction band.

Concerning the electron loss pathways, only the bulk recombination of conduction band electrons with holes in the

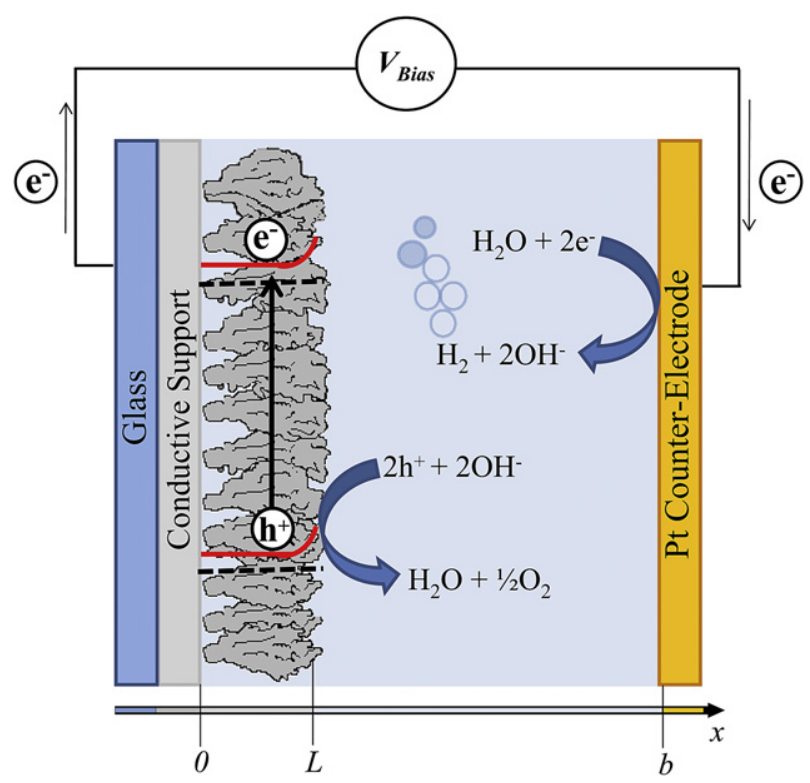

Fig. 1 - Schematic representation of the modeled PEC cell for photo-assisted water splitting. The TCO/semiconductor interface at $x=0$ and the electrolyte/platinized TCO at $x=b$ define the limits of the photoanode and the cathode, respectively. The electrolyte solution fills the pores in the photoanode with thickness $L$ and the free volume between $x=L$ and $x=b$. The dimensions of $L$ and $b$ are not in scale since $b>>$ L. valence band is taken into account. The space-charge recombination as well as the remaining recombination mechanisms mentioned above are therefore neglected [19].

The mobile charge transport can take place via diffusion, which arises from the concentration profiles, and migration, caused by a macroscopic electric field E. Effects of microscopic electric fields in the cell and screening effects, as well as Helmholtz and diffuse layers are neglected. Thus, charge transport can be described with sufficient accuracy using diffusion constants $D_{i}$ and mobilities $\mu_{i}$, connected by the Einstein relation [30]:

$D_{i}=\frac{k_{B} T}{q} \mu_{i}, \quad i=e^{-}, h^{+}$and $\mathrm{OH}^{-}$

Taking into account the previous assumptions, the proposed phenomenological model for PEC cells comprises the continuity and transport equations for the mobile species in unsteady-state and isothermal conditions. The continuity equation for the particle density $n_{i}$ is:

$-\frac{\partial j_{i}}{\partial x}+G_{i}(x)-R_{i}(x)=\frac{\partial n_{i}}{\partial t}, \quad i=e^{-}, h^{+}$and $\mathrm{OH}^{-}$

The first term on the left-hand side of the equation represents the charge carrier flux defined by the respective transport equation. The second and third terms are the generation and relaxation rates of species $i$, respectively. Both reaction kinetics are characterized by a time constant. The term on the right-hand side of the equation corresponds to carrier i concentration history. The respective continuity equations, initial and boundary conditions for the three mobile charges are presented hereafter.

\subsection{Electrons}

As described above two contributions for the electron flux, $j_{e^{-}}$, are considered: a diffusive transport of electrons within the hematite film toward the transparent conductive substrate and a migration transport driven by a macroscopic electric field across the semiconductor layer. Equation (6) relates the electron flux at any position $x$ to the gradient of electrons concentration across the semiconductor layer, $n_{e^{-}}$, by means of the electron diffusion coefficient, $D_{e^{-}}$, the electron mobility, $\mu_{e^{-}}$, and the macroscopic electric field, E:

$j_{e^{-}}=-D_{e^{-}} \frac{\partial n_{e^{-}}}{\partial x}-\mu_{e^{-}} n_{e^{-}} E$

The generation term for electrons in Equation (5) is described by the following absorption equation [30]:

$\mathrm{G}_{e^{-}}=\eta_{\text {inj }} \alpha(\lambda) I_{0} e^{-\alpha(\lambda) x}$

It is assumed that each photon, with energy $h \nu \geq E_{g}$, absorbed by the semiconductor results in the injection of one electron into its conduction band. $\alpha(\lambda)$ is the wavelengthdependent absorption coefficient, which is a property of the material; $I_{0}$ is the incident photon flux, corrected for reflection losses of the TCO glass; and $\eta_{\text {inj }}$ is the electron injection efficiency.

The electron recombination term is here represented by a generic equation based on an excess electron density 


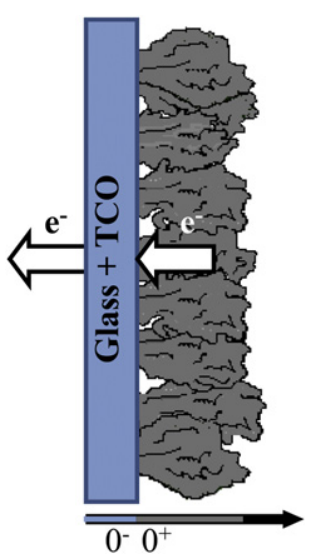

Fig. 2 - Detail of the TCO/semiconductor interface at $x=0$; $\mathrm{x}=\mathrm{0}^{+}$denotes the surface close to the semiconductor and $x=0^{-}$refers to the external surface of the TCO layer.

regarding its equilibrium value, $\Delta n_{e^{-}}=n_{e^{-}}(x, t)-n_{\text {eq }}$, and a first-order kinetics, $k_{e^{-}}=1 / \tau_{n}$ :

$R_{e^{-}}=k_{e^{-}} \Delta n_{e^{-}}$

where $\tau_{n}$ is the lifetime of the carriers before recombine and $n_{\mathrm{eq}}$ is the dark equilibrium electron density, corresponding to the match between the electron's Fermi level in the semiconductor and the redox potential of the electrolyte:

$n_{\text {eq }}=N_{\mathrm{CB}} \exp \left[-\frac{q\left(E_{\mathrm{CB}}-E_{\text {Redox }}\right)}{k_{\mathrm{B}} T}\right]$

$N_{C B}$ is the effective density of states of the respective conduction band and can be calculated by the following equation [30]:

$N_{\mathrm{CB}}=2\left(\frac{2 \pi m_{e^{-}}^{*} k_{B} T}{h^{2}}\right)$

The continuity equation, initial and boundary conditions for electrons can now be written taking all these considerations into account:

$D_{e^{-}} \frac{\partial^{2} n_{e^{-}}}{\partial x^{2}}+\mu_{e^{-}} E \frac{\partial n_{e^{-}}}{\partial x}+\mu_{e^{-}} n_{e^{-}} \frac{d E}{d x}+\eta_{\text {inj }} I_{0} \alpha e^{-\alpha x}-\frac{\left(n_{e^{-}}-n_{\mathrm{eq}}\right)}{\tau_{n}}=\frac{\partial n_{e^{-}}}{\partial t}$

\subsubsection{Initial conditions}

For $t=0$ the cell is considered to be under dark equilibrium conditions:

$t=0 \quad n_{e^{-}}(x, 0)=n_{\mathrm{eq}}$

\subsubsection{Boundary conditions}

Considering the TCO/semiconductor interface at $x=0$ as an ohmic contact with negligible energy band bending, a simple charge balance at this interface can be written in terms of the current densities at $x=0^{-}$and $x=0^{+}$, i.e. $j_{e^{-}}^{0^{-}}$and $j_{e^{-}}^{0^{+}}$, respectively:

$x=0 \quad j_{e^{-}}^{0^{-}}=j_{e^{-}}^{0^{+}}$
The current density flowing across $x=0^{+}$results from the transport of electrons by diffusion and drift across the semiconductor - Equation (6) - which, in steady-state conditions, corresponds also to the net current density, $J_{\text {cell }}$, generated by the cell under operation (see Fig. 2). This term will be analyzed and defined in more detail in Section 2.4. Thus,

$j_{e^{-}}^{0^{-}}=\frac{J_{\text {cell }}}{q}$

$j_{e^{-}}^{0+}=-\left.D_{e^{-}} \frac{\partial n_{e^{-}}}{\partial x}\right|_{x=0^{+}}-\left.\mu_{e^{-}} n_{e^{-}} E\right|_{x=0^{+}}$

At $x=L$ there are no electrons flowing from the semiconductor into the electrolyte and across it, so the respective continuity equation for electrons is only integrated between $x=0$ and $x=L$. Between the limits $x=L$ and $x=b$ only the electrolyte redox species are present.

$x=L \quad \frac{\partial n_{e^{-}}(L, t)}{\partial x}=0$

\subsection{Holes}

When the semiconductor absorbs solar radiation with enough energy to excite an electron from the valence band to the conduction band, an electron-hole pair is generated. Thus, they are generated at the same rate:

$\mathrm{G}_{h^{+}}=\mathrm{G}_{e}$

On the other hand, if a radiative recombination phenomenon is considered, a hole in the valence band reacts with an electron from the conduction band and the correspondent energy is dissipated. The recombination of a hole with a conduction band electron occurs at the same rate of the spontaneous transition of an electron from the conduction band to an unoccupied state in the valence band:

$R_{h^{+}}=R_{e^{-}}$

The respective continuity equation for holes is similar to the one defined for electrons:

$D_{h^{+}} \frac{\partial^{2} n_{h^{+}}}{\partial x^{2}}+\mu_{h^{+}} E \frac{\partial n_{h^{+}}}{\partial x}+\mu_{h^{+}} n_{h^{+}} \frac{d E}{d x}+\eta_{\text {inj }} I_{0} \alpha e^{-\alpha x}-\frac{\Delta n_{e^{-}}}{\tau_{n}}=\frac{\partial n_{h^{+}}}{\partial t}$

\subsubsection{Initial conditions}

The initial concentration of holes equals the dark equilibrium electrons density since electrons and holes are generated at the same rate:

$t=0 \quad n_{h^{+}}(x, 0)=n_{e^{-}}(x, 0)$

\subsubsection{Boundary conditions}

There are no holes flowing from the semiconductor to the TCO substrate neither from the semiconductor to the electrolyte:

$x=0 \quad \frac{\partial n_{h^{+}}(0, t)}{\partial x}=0$

$x=L \quad \frac{\partial n_{h^{+}}(L, t)}{\partial x}=0$ 


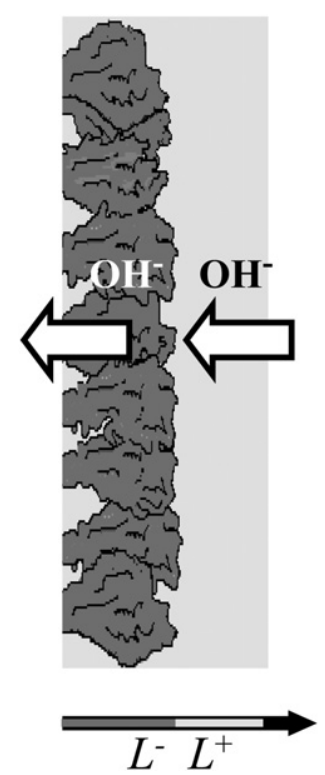

Fig. 3 - Detail of the semiconductor/electrolyte interface at $x=L$. Here, $x=L^{-}$and $x=L^{+}$denote the limit of the interface semiconductor/electrolyte at the semiconductor and electrolyte sides, respectively.

\subsection{Hydroxyl ions}

Hydroxyl ions are formed in the counter-electrode by reduction of water to hydrogen gas. Then $\mathrm{OH}^{-}$ions diffuse to the semiconductor surface where they react with holes to produce oxygen gas. However, the transport of hydroxyl ions occurs in two different regions of the PEC cell: in the pores of the mesoscopic semiconductor film (with porosity $\varepsilon_{p}$ and tortuosity $\tau$, between $x=0$ and $x=L$ ) and within the free volume between $x=L$ and the counter-electrode at $x=b$. Generation and recombination of hydroxyl ions occur only in the pores of the semiconductor, and not in the bulk electrolyte layer (with thickness $b-\mathrm{L}$ ) where only ionic transport is considered. Thus, two distinct equations should be written for this redox species.

\subsection{1. $0 \leq \mathrm{x} \leq \mathrm{L}$}

Attempting to the stoichiometry of Equations (2) and (3), the generation of two hydroxyl ions is always linked with the consumption of two electrons, while the consumption rate of hydroxyl ions equals the generation rate of holes. The charge transport is again governed by diffusion and drift:

$D_{\mathrm{OH}^{-}}^{L} \frac{\partial^{2} n_{\mathrm{OH}^{-}}^{L}}{\partial x^{2}}+\mu_{\mathrm{OH}^{-}}^{L} E \frac{\partial n_{\mathrm{OH}^{-}}^{L}}{\partial x}$

$+\mu_{\mathrm{OH}^{-}}^{\mathrm{L}} n_{\mathrm{OH}^{-}}^{L} \frac{\mathrm{dE}}{\mathrm{dx}}-\frac{1}{\varepsilon_{p}}\left(\eta_{\text {inj }} \mathrm{I}_{0} \alpha e^{-\alpha x}-\frac{\Delta n_{e^{-}}}{\tau_{e^{-}}}-\frac{\partial n_{e^{-}}}{\partial \mathrm{t}}\right)=\frac{\partial n_{\mathrm{OH}^{-}}^{L}}{\partial t}$

where

$D_{\mathrm{OH}^{-}}^{L}=\frac{\varepsilon_{p}}{\tau} D_{\mathrm{OH}^{-}}$and $\mu_{\mathrm{OH}^{-}}^{\mathrm{L}}=\frac{\varepsilon_{p}}{\tau} \mu_{\mathrm{OH}^{-}}$

are the effective diffusion and mobility constants defined for the hydroxyl ions. The superscript $L$ is included to clearly show that the region under consideration is between $x=0$ and $x=L$. The sixth term in Equation (23), $\partial n_{e^{-}} / \partial t$, is included since in unsteady-state the accumulated electrons do not participate in the redox reaction and therefore must be discounted from the generation term:

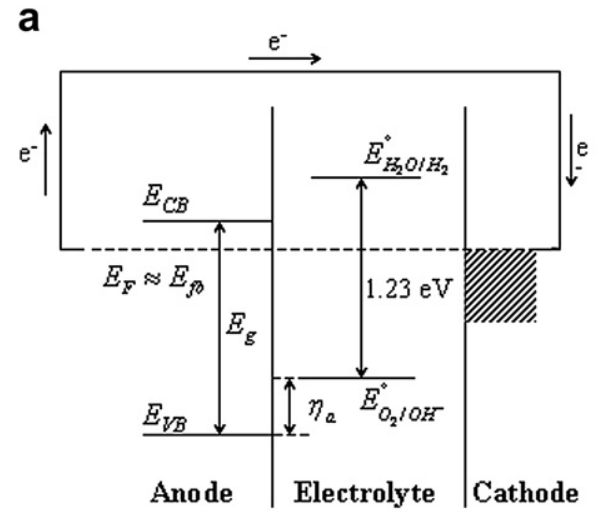

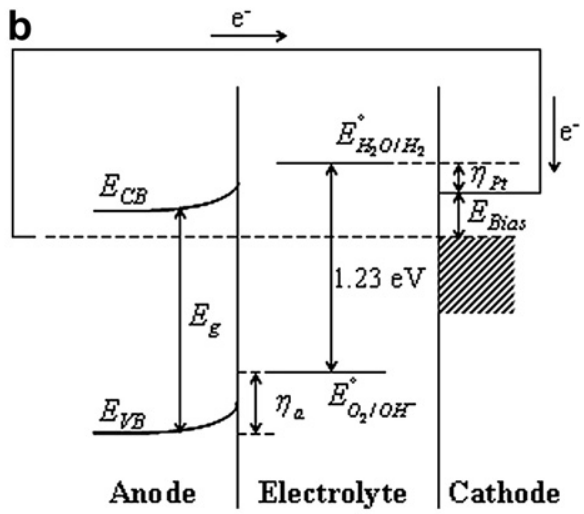

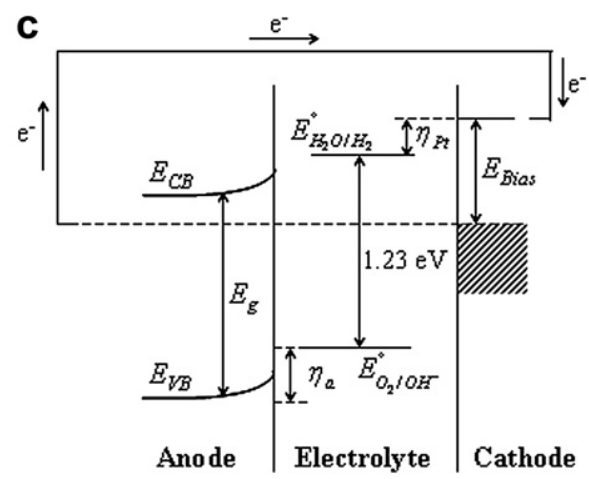

Fig. 4 - Energy diagram of PEC components with (a) no applied bias $E_{\text {Bias; }}$ (b) applied bias $E_{\text {Bias }}$ but not enough for hydrogen evolution and (c) applied bias $E_{\text {Bias }}$ enough to produce hydrogen. 
$\underbrace{\eta_{\text {inj }} \alpha(\lambda) I_{0} e^{-\alpha(\lambda) x}}_{\text {generation term }}-\underbrace{\frac{\partial n_{e^{-}}}{\partial t}}_{\text {electron accumulation }}$

2.3.2. $\mathrm{L}<\mathrm{x} \leq \mathrm{b}$

Only electrolyte exists in this region and so no ions are generated or lost by recombination with holes. So, the continuity equation is as follows:

$D_{\mathrm{OH}^{-}} \frac{\partial^{2} n_{\mathrm{OH}^{-}}^{b}}{\partial x^{2}}+\mu_{\mathrm{OH}^{-}} E \frac{\partial n_{\mathrm{OH}^{-}}^{b}}{\partial x}+\mu_{\mathrm{OH}^{-}} n_{\mathrm{OH}^{-}}^{b} \frac{\mathrm{dE}}{\mathrm{dx}}=\frac{\partial n_{\mathrm{OH}^{-}}^{b}}{\partial \mathrm{t}}$

where the superscript $b$ denotes the region between $x=L$ and $x=b$. Finally, initial and boundary conditions may now be defined for hydroxyl ions in the spatial range $0<x \leq b$.

\subsubsection{Initial conditions}

At instant $t=0$, initial concentration of the electrolyte species is assumed:

$t=0 \quad n_{\mathrm{OH}^{-}}^{\mathrm{L}}(x, 0)=n_{\mathrm{OH}^{-}}^{b}(x, 0)=n_{\mathrm{OH}^{-}}^{\text {init }}$

\subsubsection{Boundary conditions}

At the TCO/semiconductor interface only the photoinjected electrons are able to flow. Thus, the net flux of $\mathrm{OH}^{-}$is zero:

$x=0 \quad \frac{\partial n_{\mathrm{OH}^{-}}^{L}(0, t)}{\partial x}=0$

For $x=L$, corresponding to the transition between the porous material and the bulk liquid, additional considerations have to be taken into account. At this interface the concentrations of $\mathrm{OH}^{-}$ions in the liquid electrolyte filling the pores of the semiconductor and in the bulk liquid are identical:

$n_{\mathrm{OH}^{-}}^{L^{-}}=n_{\mathrm{OH}^{-}}^{L^{+}}$

Moreover, by evaluating the charge balance at the interface semiconductor/electrolyte - see Fig. 3 - the hydroxyl ions flux at $x=L^{-}$(within the porous semiconductor structure) equals the hydroxyl ions flux at $x=L^{+}$(bulk electrolyte):

$x=L \quad j_{\mathrm{OH}^{-}}^{\mathrm{L}^{-}}=j_{\mathrm{OH}^{-}}^{\mathrm{L}+}$

where:

$j_{\mathrm{OH}^{-}}^{\mathrm{L}-}=-\left.D_{\mathrm{OH}^{-}}^{L} \frac{\partial n_{\mathrm{OH}^{-}}^{L}}{\partial x}\right|_{x=L^{-}}-\left.\mu_{\mathrm{OH}^{-}}^{L} n_{\mathrm{OH}^{-}}^{L} E\right|_{x=L-}$

$j_{\mathrm{OH}^{-}}^{\mathrm{L}+}=-\left.D_{\mathrm{OH}^{-}} \frac{\partial n_{\mathrm{OH}^{-}}^{b}}{\partial x}\right|_{x=L^{+}}-\left.\mu_{\mathrm{OH}^{-}} n_{\mathrm{OH}^{-}}^{b} E\right|_{x=L^{+}}$

The total amount of the redox species contained in the semiconductor nanopores and in the free electrolyte volume remains constant during the PEC cell operation. This may be described as:

$x=b \quad \int_{0}^{L} n_{\mathrm{OH}^{-}}^{L}(x) \mathrm{d} x+\int_{L}^{b} n_{\mathrm{OH}^{-}}^{b}(x) \mathrm{d} x=n_{\mathrm{OH}^{-}}^{\text {init }} L+n_{\mathrm{OH}^{-}}^{\text {init }}(b-L)$

\subsection{Macroscopic electric field: Poisson equation}

In undoped semiconductors the number of charge carriers is determined by the properties of the material itself instead of the amount of impurities. Thus, the number of excited electrons and the number of holes are equal. For this type of semiconductors the macroscopic electric field $E$ is also related to the charge carrier densities by the Poisson's equation [26]:

$\frac{\mathrm{d}^{2} E}{\mathrm{~d} x^{2}}=\frac{q n_{\text {int }}}{\varepsilon \varepsilon_{0}} \exp \left[\frac{q E(x)}{k_{B} T}\right]$

where $n_{\text {int }}$ is the intrinsic carrier concentration given by

$n_{\text {int }}=\left(N_{\mathrm{CB}} N_{\mathrm{VB}}\right)^{1 / 2} \exp \left(-\frac{E_{g}}{2 k_{\mathrm{B}} \mathrm{T}}\right)$

where $\varepsilon$ and $\varepsilon_{0}$ are the static relative permittivity or dielectric constant of the material and the permittivity of free space, respectively. The dielectric constant describes the effective dielectric behavior of the quasi-homogeneous medium.

The model assumes that there are no unbalanced electric charges for $x \leq 0$ and $x \geq b$. Thus, the electric field vanishes exactly at these boundaries [31]:

$E(x=0)=E(x=b)=0$

\subsection{Electrolyte-platinum contact: Butler-Volmer equation}

The non-linear differential Equations (11), (19), (23) and (25) for the three charged species are integrated with the respective initial and boundary conditions. All these conditions were described and defined mathematically, but the boundary condition for electrons at $x=0$ requires a deeper analysis. In fact, it was mentioned above that the current density flowing across $x=0^{+}$results from the transport of electrons by diffusion and drift through the semiconductor - Equation (6) - which corresponds, in steady-state conditions, to the net current density, $J_{\text {cell }}$, generated by the cell under operation. This variable $J_{\text {cell }}$ corresponds to the current density that is returned to the cell via the counter-electrode and is a consequence of the external potential difference applied to the cell. At the counter-electrode, electrons take part in the water reduction reaction catalyzed by the platinum wire. Consequently, the electrolyte/platinum interface can be treated as an electrochemical half-cell and a Butler-Volmer kinetics is therefore considered [32]:

$$
\begin{aligned}
J_{\text {cell }}= & J_{0}\left[\frac{n_{\mathrm{H}_{2} \mathrm{O}}(b)}{n_{\mathrm{H}_{2} \mathrm{O}}^{\text {ref }}(b)} \exp \left(\frac{\beta n q \eta_{\mathrm{Pt}}}{k_{\mathrm{B}} \mathrm{T}}\right)\right. \\
& \left.-\frac{n_{\mathrm{H}_{2}}(b) n_{\mathrm{OH}^{-}}^{2}(b)}{n_{\mathrm{H}_{2}}^{\mathrm{ref}}(b)\left(n_{\mathrm{OH}^{-}}^{\text {ref }}\right)^{2}(b)} \exp \left(\frac{-(1-\beta) n q \eta_{\mathrm{Pt}}}{k_{\mathrm{B}} T}\right)\right]
\end{aligned}
$$

The Butler-Volmer equation defines an exponential relation between the electric current crossing the electrolyte/ platinum interface and the activation overpotential, $\eta_{\mathrm{Pt}}$, in the platinum cathode, where water is reduced to hydrogen gas with the consumption of two electrons ( $n=2$ in Equation (36)). The overpotential may be regarded as the extra voltage needed to reduce the energy barrier of the rate determining step to a value such that the electrode reaction proceeds at a desired rate. The concentrations of the species involved in such reaction have to be normalized by a reference concentration: $n_{\mathrm{H}_{2} \mathrm{O}}^{\text {ref }}, n_{\mathrm{H}_{2}}^{\text {ref }}, n_{\mathrm{OH}^{-}}^{\text {ref }}$. For dilute solutions in pure water at 
a given pressure, water concentration is virtually constant. So, if pure water at approximately atmospheric pressure is taken as the standard state for $\mathrm{H}_{2} \mathrm{O}, n_{\mathrm{H}_{2} \mathrm{O}}$ and $n_{\mathrm{H}_{2} \mathrm{O}}^{\mathrm{ref}}$ concentrations in Equation (36) are equal. Additionally, the reference concentration for hydroxyl ions is its initial concentration, while the reference hydrogen concentration is taken as the maximum solubility of hydrogen in water at 1 bar and $25{ }^{\circ} \mathrm{C}$ : $8.465 \times 10^{-5} \mathrm{~mol} \mathrm{dm}^{-3}[33$.

In what concerns the definition of the total overpotentials in the PEC cell, a thoughtful analysis needs to be made. The operation of a photoelectrochemical cell is analogous to that of a Schottky barrier and therefore the input energy of an absorbed photon is equal to the semiconductor bandgap $E_{g}$. Energy losses in the semiconductor result from the movement of electrons and holes from their point of generation to the respective electrolyte/electrode interface, and from the difference between the energy conduction band edge in the bulk and the Fermi level, $\Delta E_{F}=E_{F}(x=0)-E_{C B}$. The former phenomenon is responsible for the energy band bending $E_{B}$ observed at the electrolyte/electrode interface. In the electrolyte, only a fraction of this energy is used to originate free energy per electron for the overall cell reaction $(\Delta G)$ $n F=1.23 \mathrm{eV}$ for water decomposition). The remaining energy is lost through overpotentials at the anode and the cathode, $\eta_{a}$ and $\eta_{\mathrm{Pt}}$, respectively, and through ohmic losses, $\eta_{\text {ohmic }}$. The following energy balance can be written [11]:

$E_{\text {Bias }}+E_{g}-E_{B}-\Delta E_{F}=\frac{\Delta G}{n F}+\eta_{a}+\eta_{\mathrm{Pt}}+\eta_{\text {ohmic }}$

The term $E_{\text {Bias }}$ in the left-hand side of Equation (37) is added when an external bias applied to the PEC cell is required.

When a semiconductor is brought into contact with an electrolyte, equilibrium of the electrochemical potentials of the two phases is established by electron transfer across the interface [16]. This produces a positive space-charge layer in the semiconductor side, also called depletion layer since the region is depleted of majority charge carriers (electrons). Additionally, a charged layer is also formed in the electrolyte side, adjacent to the interface with the solid electrode - known as Helmholtz layer. However, this is not considered in the present model. Finally, the extent of band bending is given by [11]:

$E_{B}=E_{F}(x=0)-E_{f b}$

where $E_{F}$ is the Fermi level of the semiconductor and $E_{f b}$ is the semiconductor flat band potential, which reflects the energy state at which the semiconductor bands are flat (zero space charge in the semiconductor).

The anodic overpotential is considered as the difference between the valence band edge potential and the oxidation potential of water [34]:

$\eta_{a}=E_{\mathrm{VB}}-E_{\mathrm{O}_{2} / \mathrm{OH}^{-}}$

where the oxidation potential of water is directly related to the water reduction potential by the standard free enthalpy for water splitting $(1.23 \mathrm{eV})$ :

$E_{\mathrm{O}_{2} / \mathrm{OH}^{-}}=1.23-\mathrm{E}_{\mathrm{H}_{2} \mathrm{O} / \mathrm{H}_{2}}$

In its turn, the electrochemical potential of water reduction to hydrogen at the platinum electrode is given by the Nernst equation:
$E_{\mathrm{H}_{2} \mathrm{O} / \mathrm{H}_{2}}=E_{\mathrm{H}_{2} \mathrm{O} / \mathrm{H}_{2}}^{\circ}-\frac{k_{\mathrm{B}} \mathrm{T}}{2 q} \ln \frac{n_{\mathrm{H}_{2}}(b) n_{\mathrm{OH}^{-}}^{2}(b)}{n_{\mathrm{st}}^{3}}$

The standard concentration $n_{\mathrm{St}}$ is here defined as a refer-

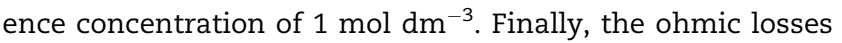
through the electrode area A may be written in terms of an ohmic resistance, $R_{\text {ohmic: }}$ :

$\eta_{\text {ohmic }}=J_{\text {cell }} A R_{\text {ohmic }}$

At this moment all the terms of Equation (37) can be evaluated and thus it can be solved in order to the cathode overpotential, $\eta_{\mathrm{Pt}}$. Then, the resulting equation can be substituted in the Butler-Volmer equation in order to find the net current density $J_{\text {cell }}$ at a defined applied external bias $E_{\text {Bias }}$.

The parameter $E_{\text {Bias }}$ is of great importance since it determines the possibility of hydrogen evolution takes place or not. In fact, for efficient water cleavage, the conduction band of the n-type semiconductor needs to be positioned at a more negative potential than the reduction potential of water, i.e. $E_{\mathrm{CB}}<E_{\mathrm{H}_{2} \mathrm{O} / \mathrm{H}_{2}}^{\circ}$, while the valence band needs to be positioned at a more positive potential than the oxidation reaction, i.e. $E_{\mathrm{VB}}>E_{\mathrm{O}_{2} / \mathrm{OH}^{-}}^{\circ}$. However, for a hematite porous film, the conduction band potential does not fulfill the required potential position and so an additional bias is needed. Varying the value of the applied bias, three different situations should be considered: i) $E_{\text {Bias }}=0$, meaning that no bias is applied; ii) the applied bias is higher than zero but not sufficiently high to produce hydrogen evolution and iii) the applied bias is high enough to produce hydrogen. The energy diagrams for these three situations are sketched in Fig. 4 and for each case the energy balance is showed.

At high intensity illumination the semiconductor potential is driven toward its flat band potential and in the case of zero bias - Fig. 4(a) - the band bending is negligible $-E_{B} \approx 0$ since $E_{f b} \approx E_{F}$. When no bias is applied, Equation (37) reduces to:

$\eta_{\mathrm{Pt}}=E_{g}-\left(E_{f b}-E_{C B}\right)-\frac{\Delta G}{n F}-\eta_{a}-\eta_{\text {ohmic }}$

Since there is no bias, the system is not able to produce hydrogen gas and so the water reduction potential equals the standard potential of $-0.828 \mathrm{~V}$ in alkaline environment and at $25^{\circ} \mathrm{C}$ :

$\eta_{a}=E_{\mathrm{VB}}-\left(1.23-E_{\mathrm{H}_{2} \mathrm{O} / \mathrm{H}_{2}}^{\circ}\right)$

Equation (44) can also be used for the case when an anodic bias is applied but it is not sufficient to produce hydrogen Fig. 4(b). In this case the band bending cannot be neglected:

$\eta_{\mathrm{Pt}}=E_{\text {Bias }}+E_{g}-\left(E_{F}-E_{f b}\right)-\left(E_{F}-E_{\mathrm{CB}}\right)-\frac{\Delta G}{n F}-$
$\left[E_{\mathrm{VB}}-\left(1.23-E_{\mathrm{H}_{2} \mathrm{O} / \mathrm{H}_{2}}^{\circ}\right)\right]-\eta_{\text {ohmic }}$

For the generic case represented in Fig. 4(c), the overpotential of cathode is given by:

$\eta_{\mathrm{Pt}}=E_{\mathrm{Bias}}+E_{g}-\left(E_{\mathrm{F}}-E_{f b}\right)-\left(E_{\mathrm{F}}-E_{\mathrm{CB}}\right)-\frac{\Delta G}{n F}-$
$\left[E_{\mathrm{VB}}-\left(1.23-E_{\mathrm{H}_{2} \mathrm{O} / \mathrm{H}_{2}}^{\circ}+\frac{k_{\mathrm{B}} \mathrm{T}}{2 q} \ln \left(n_{\mathrm{H}_{2}} n_{\mathrm{OH}^{-}}^{2}\right)\right)\right]-\left(J_{\text {cell }} \mathrm{AR}_{\text {ohmic }}\right)$

For the cases illustrated in Fig. 4(a) and (b), since there is no hydrogen gas evolution, the second term of the Butler-Volmer 
equation - Equation (36) - is zero, becoming $J_{\text {cell }}=J_{0} \exp \left(\frac{2 \beta q}{k_{\mathrm{B}} \mathrm{T}} \eta_{\mathrm{Pt}}\right)$. The Butler-Volmer equation assumes its original form given in Equation (36) only for the case represented in Fig. 4(c).

\subsection{Dimensionless equations}

The model variables were made dimensionless with respect to electron parameters and to the thickness of the semiconductor L. Changing for dimensionless variables and introducing suitable dimensionless parameters, the continuity equations for the three charge carrier species and the respective initial and boundary conditions become:

$D_{e^{-}}^{*} \frac{\partial^{2} n_{e^{-}}^{*}}{\partial x^{* 2}}+\mu_{e^{-}}^{*} \zeta\left(E^{*} \frac{\partial n_{e^{-}}^{*}}{\partial x^{*}}+n_{e^{-}}^{*} \frac{d E^{*}}{\partial x^{*}}\right)+D a \cdot e^{-\gamma x *}-\Phi^{2}\left(n_{e^{-}}^{*}-n_{\mathrm{eq}}^{*}\right)=\frac{\partial n_{e^{-}}^{*}}{\partial \theta}$

$\theta=0 \quad n_{e^{-}}^{*}\left(x^{*}, 0\right)=n_{\mathrm{eq}}^{*}$

$x^{*}=0 \quad J_{\text {cell }}^{*}=\frac{n_{\text {ref }} D_{\text {ref }} q}{J_{0} L}\left(-\left.D_{e^{-}}^{*} \frac{\partial n_{e^{-}}^{*}}{\partial x^{*}}\right|_{x^{*}=0^{+}}\right)$

$-\frac{\mu_{\text {ref }} n_{\text {ref }} E_{\text {ref }} q}{J_{0}}\left(\left.\mu_{e^{-}}^{*} n_{e^{-}}^{*} E^{*}\right|_{x^{*}=0^{+}}\right)$

$x^{*}=1 \quad \frac{\partial n_{e^{-}}^{*}}{\partial x^{*}}=0$

$D_{h^{+}}^{*} \frac{\partial^{2} n_{h^{+}}^{*}}{\partial x^{* 2}}+\mu_{h^{+}}^{*} \zeta\left(E^{*} \frac{\partial n_{h^{+}}^{*}}{\partial x^{*}}+n_{h^{+}}^{*} \frac{d E^{*}}{\partial x^{*}}\right)+D a \cdot e^{-\gamma x^{*}}$

$-\Phi^{2}\left(n_{e^{-}}^{*}-n_{\mathrm{eq}}^{*}\right)-\frac{\partial n_{e^{-}}^{*}}{\partial \theta}=\frac{\partial n_{h^{+}}^{*}}{\partial \theta}$

$\theta=0 \quad n_{h^{+}}^{*}\left(x^{*}, 0\right)=n_{\mathrm{eq}}^{*}$

$x^{*}=0 \quad \frac{\partial n_{h^{+}}^{*}}{\partial x^{*}}=0$

$x^{*}=1 \quad \frac{\partial n_{h^{+}}^{*}}{\partial x^{*}}=0$

$D_{\mathrm{OH}^{-}}^{L *} \frac{\partial^{2} n_{\mathrm{OH}^{-}}^{L^{*}}}{\partial x^{* 2}}+\mu_{\mathrm{OH}^{-}}^{L *} \zeta\left(E^{*} \frac{\partial n_{\mathrm{OH}^{-}}^{L^{*}}}{\partial x^{*}}+n_{\mathrm{OH}^{-}}^{L^{*}} \frac{\mathrm{d} E^{*}}{\partial x^{*}}\right)$

$-\frac{1}{\varepsilon_{p}}\left[D a \cdot e^{-\gamma X *}-\Phi^{2}\left(n_{e^{-}}^{*}-n_{\mathrm{eq}}^{*}\right)-\frac{\partial n_{e^{-}}^{*}}{\partial \theta}\right]=\frac{\partial n_{\mathrm{OH}^{-}}^{L^{*}}}{\partial \theta}$

$D_{\mathrm{OH}^{-}}^{*} \frac{\partial^{2} n_{\mathrm{OH}^{-}}^{b *}}{\partial x^{* 2}}+\mu_{\mathrm{OH}^{-}}^{*} \zeta\left(E^{*} \frac{\partial n_{\mathrm{OH}^{-}}^{b *}}{\partial x^{*}}+n_{\mathrm{OH}^{-}}^{b *} \frac{d E^{*}}{\partial x^{*}}\right)=\frac{\partial n_{\mathrm{OH}^{-}}^{b *}}{\partial \theta}$

$\theta=0 \quad n_{\mathrm{OH}^{-}}^{L *}\left(x^{*}, 0\right)=n_{\mathrm{OH}^{-}}^{b *}\left(x^{*}, 0\right)=n_{\mathrm{OH}^{-}}^{* \text { init }}$

$x^{*}=1 \quad j_{\mathrm{OH}^{-}}^{* 1-}=j_{\mathrm{OH}^{-}}^{* 1+}$ and $n_{\mathrm{OH}^{-}}^{* 1-}=n_{\mathrm{OH}^{-}}^{* 1+}$

$x^{*}=b / L \quad \int_{0}^{1} n_{\mathrm{OH}^{-}}^{* L}\left(x^{*}\right) d x^{*}+\int_{1}^{b / L} n_{\mathrm{OH}^{-}}^{* b}\left(x^{*}\right) d x^{*}$

$=n_{\mathrm{OH}^{-}}^{* \text { init }}+n_{\mathrm{OH}^{-}}^{* \text { init }}(b / L-1)$

where

$$
\begin{aligned}
& n_{i}^{*}=\frac{n_{i}}{n_{\text {ref }}}, \quad x^{*}=\frac{x}{L}, \quad D_{i}^{*}=\frac{D_{i}}{D_{\text {ref }}}, \quad \mu_{i}^{*}=\frac{\mu_{i}}{\mu_{\text {ref }}}\left(i=e^{-}, h^{+}, \quad \mathrm{OH}^{-}\right), \\
& E^{*}=\frac{E}{E_{\text {ref }}}, \quad \theta=\frac{t}{\tau}, \quad \tau_{\text {ref }}=\frac{L^{2}}{D_{\text {ref }}}, \quad J_{\text {cell }}^{*}=\frac{J_{\text {cell }}}{J_{0}}, \zeta=\frac{\mu_{\text {ref }} E_{\text {ref }} L}{D_{\text {ref }}}, \\
& D a=\frac{L^{2} \eta_{\text {inj }} \alpha I_{0}}{D_{\text {ref }} n_{\text {ref }}}, \quad \gamma=\alpha L \text { and } \Phi=L \sqrt{\frac{k_{e^{-}}}{D_{\text {ref }}}} .
\end{aligned}
$$

\subsection{Numerical solution strategy}

To simulate a PEC cell it is necessary to solve Equations (47), (51), (55) and (56) with the respective boundary and initial conditions. In order to apply the boundary condition at $x^{*}=0$ for the electrons, for a given value of $E_{\text {Bias, }}$ Equations (43) or (46) should be substituted in Equation (36) to obtain the current density in the external circuit. The partial differential equations were spatially discretized using the finite differences method. The time integration was accomplished using LSODA [35], a numerical package developed at the Lawrence Livermore National Laboratory. To compute the I-V characteristic curves, the equations were solved for steady-state conditions (whenever the time derivative was smaller than a pre-defined value).

\section{Results and discussion}

\subsection{I-V characteristic of an undoped hematite electrode based-PEC cell}

The proposed model was used to compute the I-V characteristic curves of a mesoporous undoped hematite photoanode studied by Cesar et al. [36] and compared with the corresponding experimental results obtained by these authors. The hematite sample was prepared by atmospheric pressure chemical vapor deposition (APCVD) and the deposition time was $1 \mathrm{~min}$ [36]. The experimental current density evolution versus potential was measured using a $1 \mathrm{M} \mathrm{NaOH}$ electrolyte solution ( $\mathrm{pH}$ 13.6), at $50 \mathrm{mV} \mathrm{s}^{-1}$, under 1 sun simulated back-illumination (AM $1.5 \mathrm{G}$, $100 \mathrm{~mW} \mathrm{~cm}^{-2}$ ). The experimental data extracted from the work by Cesar et al. [36] were used for simulation purposes and are compiled in Table 1, together with other model parameters obtained in the literature. The experimental and simulated I-V curves were fitted adjusting parameters $R_{\text {ohmic }}$ and $\eta_{\text {inj. }}$.

Fig. 5 compares the experimental data with the corresponding simulated I-V curve. As it can be realized from this figure, the model agrees quite well with the experimental data, especially for voltages lower than 1.6 V. For higher values of voltage the electrocatalytic oxygen evolution starts by the reaction of holes with the $\mathrm{OH}^{-}$ions in the electrolyte. This phenomenon explains the accentuated current density increase noticed in both characteristic curves for voltages higher than 1.6-1.7 V.

Considering a "cappuccino" like PEC cell, described elsewhere [38], the illumination of the photoanode can be made through the front-side (via the electrolyte/hematite interface) or through the back-side (via the substrate/hematite interface). Front- and back-side illumination experiments may be used to distinguish between electron and hole transport limitations, as discussed by Cesar et al. [36]. However, this conclusion can only be taken if the film thickness is larger than the reciprocal value of the absorption coefficient. For 
Table 1 - Simulator input and fitting parameters. The values extracted from the literature are indexed to the corresponding reference and the model values fitted to the experimental I-V curve are identified by "Fit".

\begin{tabular}{|c|c|c|}
\hline Parameter & Value & Reference \\
\hline $\mathrm{C}_{\mathrm{OH}^{-}}^{\text {init }}$ & $1 \mathrm{M}$ & {$[36]$} \\
\hline$D_{e^{-}}$ & $4.07 \times 10^{-4} \mathrm{~cm}^{2} \mathrm{~s}^{-1}$ & [30] \\
\hline$D_{h^{+}}$ & $1.25 \times 10^{-4} \mathrm{~cm}^{2} \mathrm{~s}^{-1}$ & [30] \\
\hline $\mathrm{D}_{\mathrm{OH}^{-}}$ & $5.26 \times 10^{-5} \mathrm{~cm}^{2} \mathrm{~s}^{-1}$ & [37] \\
\hline A & $0.5 \mathrm{~cm}^{2}$ & [29] \\
\hline L & $63 \mathrm{~nm}$ & [36] \\
\hline$b$ & $\sim 1 \mathrm{~cm}$ & [36] \\
\hline$\varepsilon_{p}$ & 0.63 & [36] \\
\hline$\tau$ & 1.0 & [36] \\
\hline$N_{C B}$ & $1.0 \times 10^{19} \mathrm{~cm}^{-3}$ & [30] \\
\hline$\tau_{n}$ & $0.22 \mathrm{~ns}$ & [36] \\
\hline$\eta_{i n j}$ & 0.93 & Fit \\
\hline$\alpha(550 \mathrm{~nm})$ & $8.5 \times 10^{4} \mathrm{~cm}^{-1}$ & [36] \\
\hline$I_{0}$ & $1.50 \times 10^{17} \mathrm{~cm}^{-2} \mathrm{~s}^{-1}$ & [36] \\
\hline$\beta$ & 0.50 & [37] \\
\hline$J_{0}$ & $1.0 \times 10^{-4} \mathrm{~mA} \mathrm{~cm}^{-2}$ & [37] \\
\hline$E_{C B}$ & $-0.5 \mathrm{~V}$ & [3] \\
\hline$E_{\mathrm{VB}}$ & $1.7 \mathrm{~V}$ & [3] \\
\hline$E_{f b}$ & $-0.43 \mathrm{~V}$ & [27] \\
\hline $\mathrm{E}_{\mathrm{H}_{2} \mathrm{O} / \mathrm{H}_{2}}^{\circ}$ & $-0.828 \mathrm{~V}$ & [3] \\
\hline $\mathrm{E}_{\mathrm{O}_{2} / \mathrm{OH}^{-}}$ & $0.402 \mathrm{~V}$ & [3] \\
\hline$\Delta G / n F$ & $1.23 \mathrm{~V}$ & [3] \\
\hline$R_{\text {ohmic }}$ & $50 \Omega$ & Fit \\
\hline $\mathrm{T}$ & $298 \mathrm{~K}$ & [29] \\
\hline $\mathrm{pH}$ & 13.6 & [36] \\
\hline
\end{tabular}

undoped samples of hematite, back-side illumination (Fig. 5) always gives higher photocurrent than front-side illumination (Fig. 6). This indicates that the rate-limiting step in undoped $\mathrm{Fe}_{2} \mathrm{O}_{3}$ samples is the electron transport, in contrast to Sidoped hematite photoanodes where hole transport is found to be rate-limiting $[39,40]$. The front-side illumination

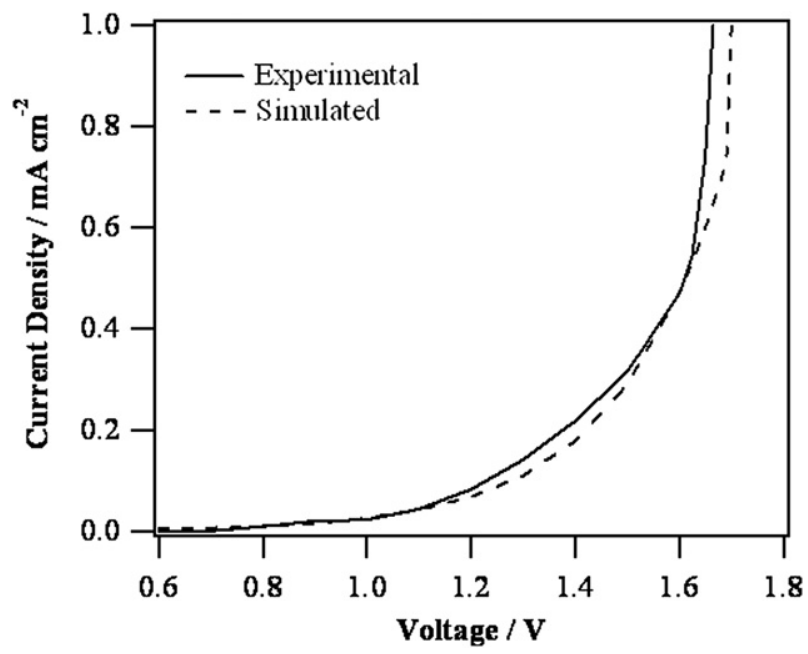

Fig. 5 - Experimental (solid line) and simulated (dashed line) I-V characteristic for the system described in Table 1 $\left(\eta_{\text {inj }}=0.93\right)$, under 1 sun back-illumination. The experimental curve was taken from Ref. [36], Fig. 6, dashed curve labeled " 1 'bc no si".

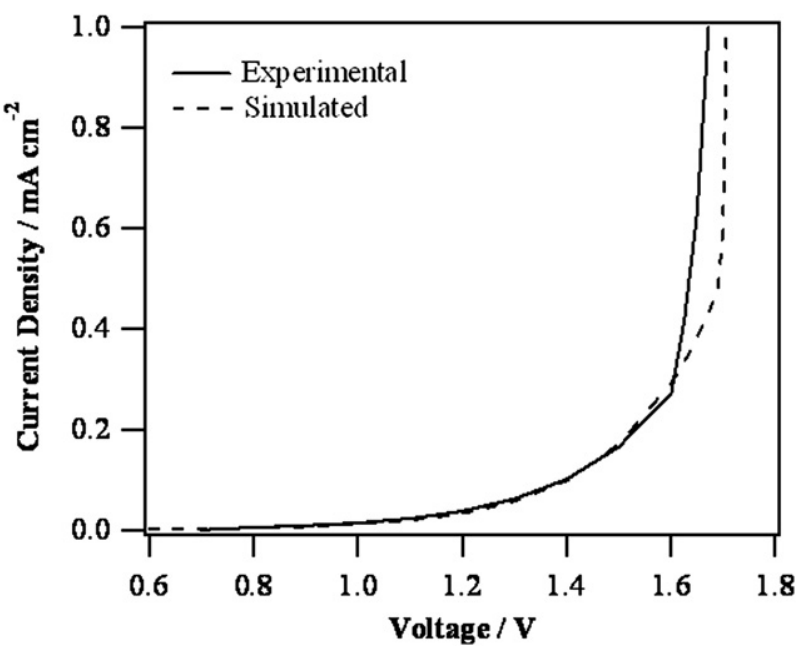

Fig. 6 - Experimental (solid line) and simulated (dashed line) I-V characteristics for a PEC cell characterized by parameters given in Table $1\left(\eta_{\text {inj }}=0.90\right)$, under 1 sun frontside illumination. The experimental curve was taken from Ref. [36], Fig. 6, solid curve labeled as “1' no Si”.

experiment was also simulated in the present work, where Equation (7) was replaced by the corresponding one for frontside irradiation:

$G_{e^{-}}=\eta_{\text {inj }} \alpha(\lambda) I_{0} e^{-\alpha(\lambda)(L-x)}$

being $L$ the hematite layer thickness. The fitted injection efficiency obtained was $90 \%$.

Fig. 7 shows the simulated steady-state electron density profiles across the $63 \mathrm{~nm}$-thick of the hematite film at $1.23 \mathrm{~V}$ and under 1 sun back-side illumination. Since the experiment was performed with back-side illumination (see scheme in Fig. 1), most electrons were generated near the glass substrate due to the exponential absorption behavior Equation (7). Thus, the maximum electron density gradient

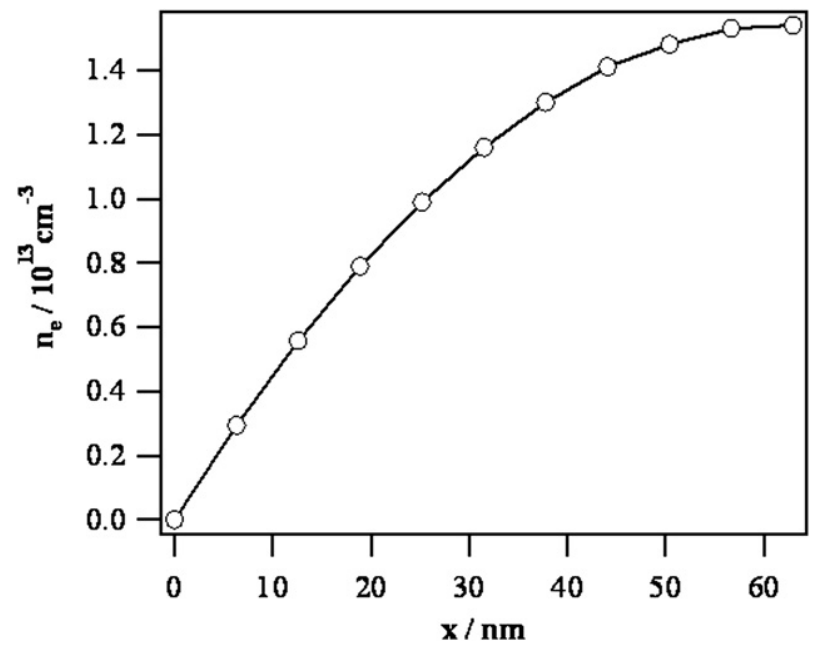

Fig. 7 - Simulated electron density profiles for the system characterized by parameters given in Table 1 under 1 sun back-side illumination at $1.23 \mathrm{~V}$. 


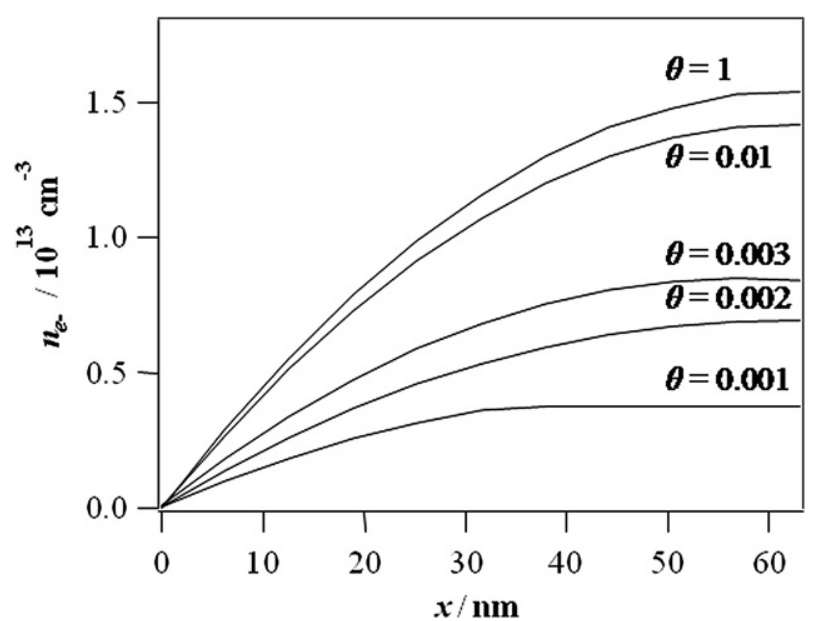

Fig. 8 - Transient electron density profiles for the system characterized by parameters given in Table 1 under 1 sun back-illumination at $1.23 \mathrm{~V}$. Density profiles are presented as a function of dimensionless time $\theta$.

is observed for axial position $x=0$. Under these conditions, the hematite layers closest to the current collector (TCO) contribute mostly to the electron flux toward the external circuit. This fact is related to high concentration gradients and then diffusion transport, limiting the electron recombination kinetics.

Fig. 8 shows the evolution of the transient electron density profiles of the undoped hematite film at $1.23 \mathrm{~V}$ and under 1 sun back-side illumination before reaching the steady-state condition. The electron density profiles during the first micrometers of the hematite film is characterized by a steady increase, becoming a flat profile towards $x=L$. For higher values of $\theta$, the electron density profile tends to the steadystate condition (shown in Fig. 7).

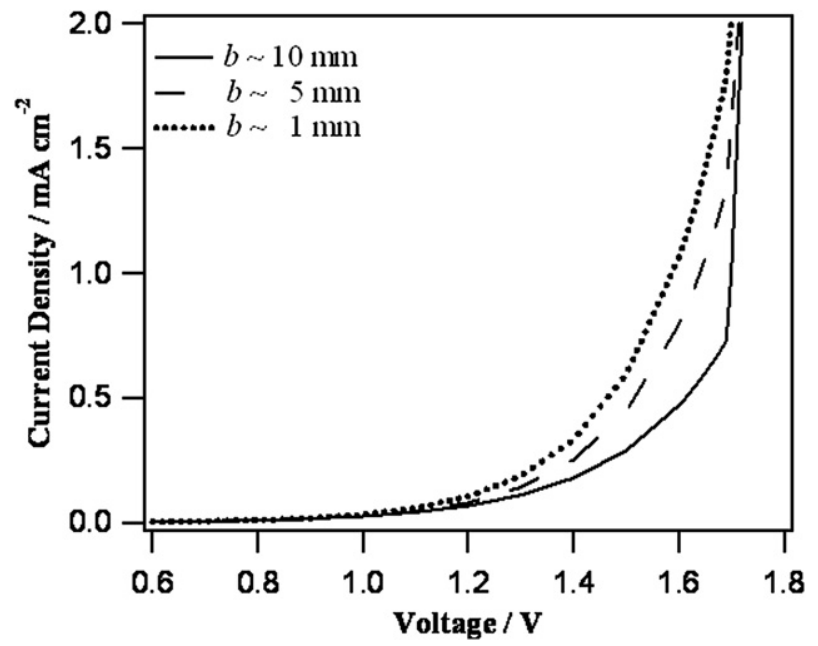

Fig. 9 - Effect of the electrolyte layer thickness in the photocurrent density produced by the PEC cell system under study. The solid curve $(b \sim 10 \mathrm{~mm})$ is the reference case presented in Table 1 and in Fig. 5.

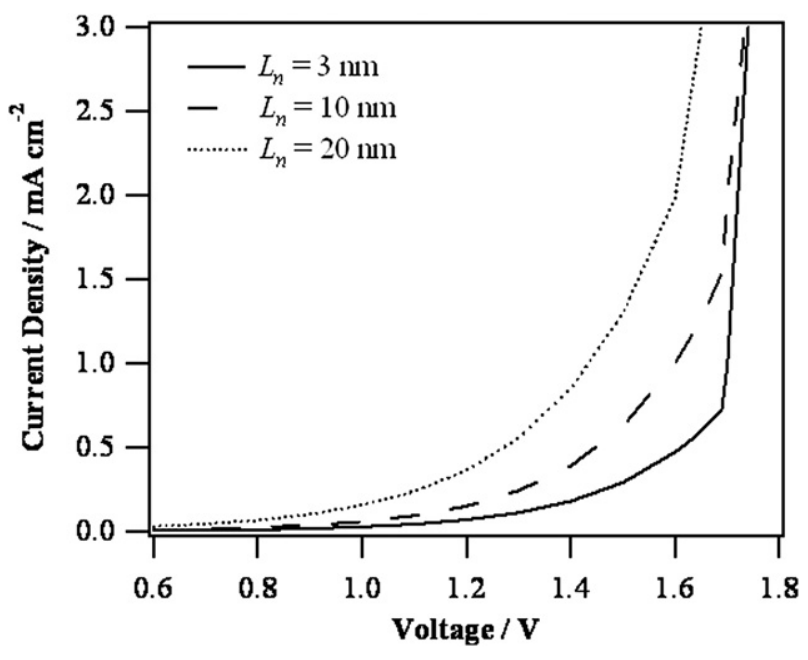

Fig. 10 - Effect of the diffusion length value in the photocurrent density produced by the PEC cell system under study. The solid curve for $L_{n}=3$ is the reference case presented in Table 1 and in Fig. 5.

\subsection{Effect of the electrolyte layer thickness}

As mentioned above, the photoelectrochemical characterization was performed in a PEC cell configuration known as "cappuccino" [36]. The illumination of the photoanode is done through an uncoated fused silica window pressed against an o-ring by a metallic window part. A conductive uncoated area of the photoanode sample is clipped onto a titanium contact. This clip can rotate to facilitate front- and back-side illumination. The counter-electrode, usually a platinum wire is placed at a distance of $\sim 10 \mathrm{~mm}$ from the photoanode. The space between the two electrodes is filled with the electrolyte solution. Consequently, the hydroxyl ions formed at the counter-electrode have to diffuse a pathway of around $10 \mathrm{~mm}$

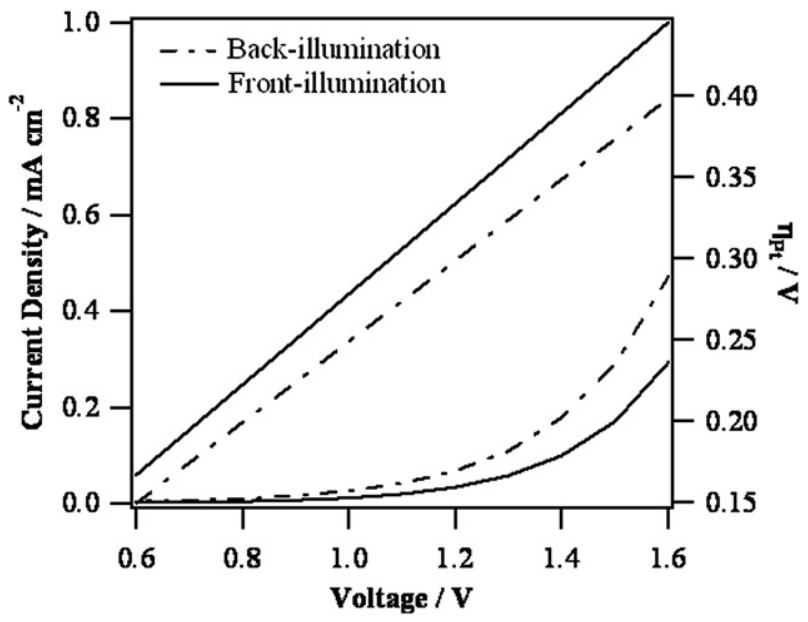

Fig. 11 - Relation between the characteristic curves with the correspondent platinum overpotentials at the counterelectrode for back- (dashed line) and front-side illumination (solid line). 


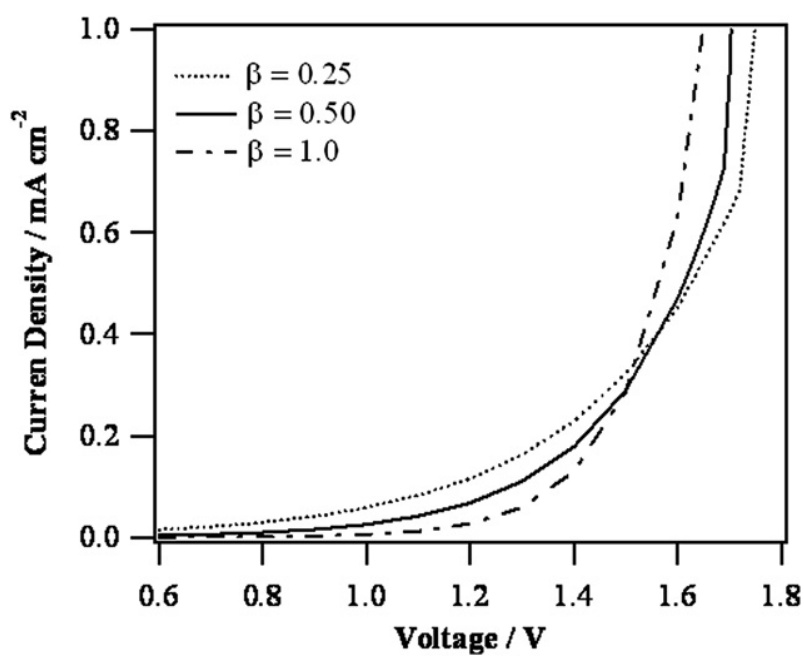

Fig. 12 - Effect of the transfer coefficient on the symmetry of the current-overpotential curves for the system under study. Again, the solid curve for $\beta=0.5$ is the reference case presented in Table 1 and in Fig. 5.

until reaching the photoanode, where water oxidation occurs. This distance is $b-\mathrm{L}$ in Fig. 1 ( $\mathrm{L}$ is almost negligible when compared with $b$ and so from now on $b-L$ will be considered approximately equal to $b$ ). Thus, by changing the thickness of the free electrolyte, the resistance that ions $\mathrm{OH}^{-}$ions have to face crossing the electrolyte layer is also different. In fact, diminishing the value of $b$, potential losses in the electrolyte are lower and the photocurrent density increases. From Fig. 9 it can be noticed that the increase in the photocurrent density is more pronounced when the electrolyte thickness decreases from $10 \mathrm{~mm}$ to $5 \mathrm{~mm}$ than from $5 \mathrm{~mm}$ to $1 \mathrm{~mm}$. This may indicate that the reduction of the electrolyte thickness to values lower than $1 \mathrm{~mm}$ is not that worthy for the photocurrent density response.

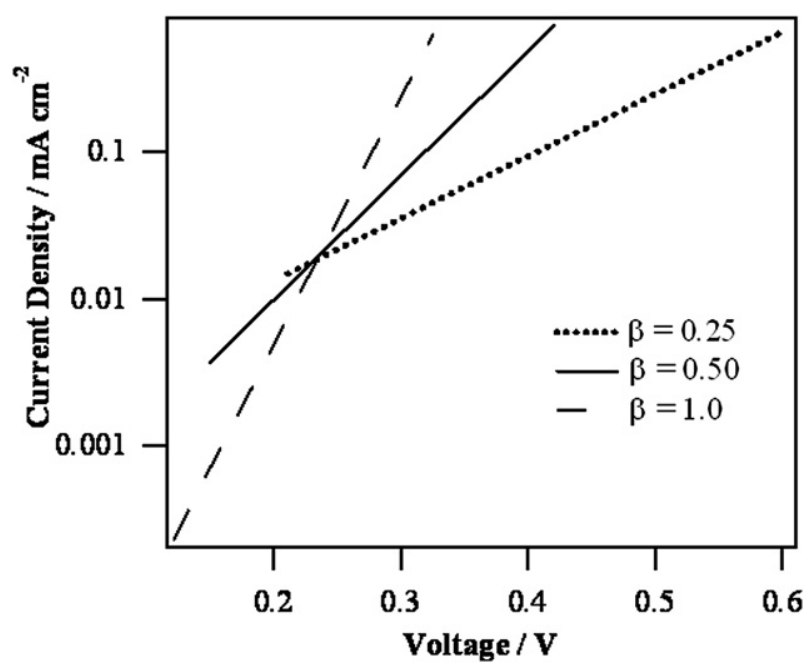

Fig. 13 - Logarithmic representation of the current density versus overpotential for the platinum counter-electrode and for high values of $\eta_{\mathrm{Pt}}-$ Tafel plots.

\subsection{Effect of the hole diffusion length}

The low values of photocurrent observed for the system under study are due to a low water oxidation rate by surface trapped holes [41] and a short hole diffusion length $L_{n}$ in hematite photoelectrodes $\left(L_{n}=\sqrt{D_{h^{+}} \tau_{n}}\right.$, being $D_{h^{+}}$and $\tau_{n}$ the diffusion coefficient and lifetime of the holes, respectively). In this present case $L_{n}=3 \mathrm{~nm}$, which implies that most holes are created in the semiconductor bulk and will recombine with electrons before having reached the surface [38]. Increasing the hole diffusion length allows a significant increase in the photocurrent density - Fig. 10 - and, consequently, in the hydrogen production by the PEC cell. However, undoped hematite shows a hole diffusion length of only 2-4 $\mathrm{nm}$ since the photon penetration depth at visible wavelengths in hematite $\left(\alpha^{-1}=118 \mathrm{~nm}\right.$ at $\left.\lambda=550 \mathrm{~nm}\right)$ causes the majority of photogenerated carriers to be produced at a distance of ca. $100 \mathrm{~nm}$ from the semiconductor liquid junction [42]; the recombination time constants of the photogenerated carriers are on the order of $10 \mathrm{ps}$ [43]; and the minority charge carrier has a poor mobility (maximum of $0.2 \mathrm{~cm}^{2} \mathrm{~V}^{-1} \mathrm{~s}^{-1}$ ) [44]. Thus, it is almost impossible to achieve the photocurrent values shown in Fig. 10 for diffusion lengths higher than $10 \mathrm{~nm}$, which explains the need of doping hematite. In fact, the performance of these photoanodes is significantly improved by doping with silicon [6].

\subsection{Effect of the Butler-Volmer kinetics}

The potential of the electrodes affects strongly the kinetics of reactions occurring on their surface. As explained before, this current-potential dependence is described by the Butler-Volmer equation. In electrochemical reactions activation losses take place, which are caused by the slowness of the reactions occurring on the surface of the electrodes. Once an electrochemical reaction is initiated, the concentration of the species taking part in the reaction at the electrode/electrolyte interface will normally be different than those in the bulk of the solution. Thus, concentration profiles arise due to effects of simple diffusion and/or effects of migration in the electric field at or near the electrode. A point to holdback at this stage is that the transport to or from the electrode surface of the species involved in the electrochemical process may not be sufficiently fast to maintain the same concentration at the electrode surface as in the bulk. If transport processes are rapid compared to charge transfer, then bulk concentration and electrode surface concentration are similar. On the other hand, when there is a considerable current flowing, the electrode surface concentration is lower than the bulk concentration and thus the potential must be increased in order to maintain the desired current density [45]. This increased potential is in fact called overpotential, here labeled as $\eta_{\mathrm{Pt}}$. By increasing the current density, more voltage is needed to be sacrificed in order to maintain those values of current. The platinum overpotential also increases with the applied voltage - Fig. 11. The present model allows to predict the platinum overpotential occurring at each specific case and how it evolves through the current-voltage characteristic.

The transfer coefficient $\beta$ that appears in the Butler-Volmer equation depends on the symmetry of the activation barrier and, for that reason, is also known as symmetry parameter. Its 
value expresses how the change in the electrical potential across the reaction interface changes the sizes of the forward versus reverse activation barrier. For $\beta=0.5$ the anodic and cathodic branches of the total current will be symmetrical. However, for $\beta$ value near unity the cathodic branch rises much more steeply than the anodic branch, whereas for $\beta$ near zero the inverse is found. The transfer coefficient depends on the reaction involved and on the electrode materials [46]. Nevertheless, for the system under analysis the variation of $\beta$, which can be obtained e.g. by using different materials, have a little impact on the performance of the PEC cell - Fig. 12.

As overpotentials become larger than about $50-100 \mathrm{mV}$, the second term of Equation (36) becomes negligible, meaning that the forward-reaction direction dominates. Hence, simple exponential relationships between current density and overpotential are obtained, i.e. the overpotential can be considered as logarithmically dependent on the current density. The Butler-Volmer equation simplifies to:

$J_{\text {cell }}=J_{0} \exp \left(\frac{\beta n q}{k_{\mathrm{B}} \mathrm{T}} \eta_{\mathrm{Pt}}\right)$

Equation (61) is known as the Tafel equation and plotting ln ( $\left.U_{\text {cell }}\right)$ versus $\eta_{\mathrm{Pt}}$ one obtains a straight line. Therefore, the approximation to the Tafel equation is valid. The slope of a Tafel plot is related to the transfer coefficient $\beta$ accordingly to: slope $=\beta n q /\left(k_{B} T\right)$ [32] (Fig. 13).

\section{Conclusions}

A transient phenomenological model of a PEC cell using an undoped hematite photoanode was developed. The model results were critically compared with the experimental I-V characteristics and very good agreement was observed.

This model allowed to study underlying mechanisms of the PEC cell. Steady-state electron density profiles for backillumination were obtained, suggesting that most electrons are generated near the current collector. In this part of the cell, higher concentration gradients were observed and then enhanced electron diffusion rates, which in its turn limits electron recombination kinetics. Front- and back-side illumination experiments (via the electrolyte/hematite and via the substrate/hematite interfaces, respectively) were simulated in order to distinguish between electron and hole transport limitations. For undoped samples of hematite, back-side illumination showed higher photocurrent than front-side illumination.

The influence of several operation conditions was simulated and namely thickness of free electrolyte, hole diffusion length and Butler-Volmer kinetics. By decreasing the thickness of the free electrolyte, the ionic mass transfer in that region is lower and the photocurrent density increases. The increase of hole diffusion length allows a significant increase in the photocurrent density and, consequently, in the hydrogen production by the PEC cell. Even though the simulation results showed considerable photocurrent densities for $L_{n}>10 \mathrm{~nm}$ using undoped hematite samples, it is almost impossible to achieve hole diffusion lengths in that range due to structural limitations. This fact explains the need of doping hematite photoanodes.

Finally, the reduction of water at the platinum counter-electrode was described based on the Butler-Volmer equation. The present model allows obtaining the platinum overpotential as a function of the observed current density. The effect of the transfer coefficient was assessed using the simulator. The results showed, for the system under analysis, that this variation causes little effect in the performance of the PEC cell.

\section{Acknowledgments}

This research has been funded by the European Commission (Project NanoPEC, contract number 227179) and the Portuguese Foundation for Science and Technology (Project PTDC/ EQU-EQU/107990/2008). L. Andrade and T. Lopes are grateful to the Portuguese Foundation for Science and Technology (FCT) for their PhD grants (references: SFRH/BD/30464/2006 and SFRH/BD/62201/2009, respectively). H. Aguilar Ribeiro acknowledges the Programa Ciência 2007.

\section{Nomenclature}

A

$b$

$C_{i}$

$D_{i}$

$D_{i}^{b}$

$D_{i}^{L}$

$D_{\text {ref }}$

E

$E_{\text {Bias }}$

$E_{B}$

$E_{\mathrm{CB}}$

$E_{f b}$

$E_{g}$

$E_{F}$

$\mathrm{E}_{\mathrm{H}_{2} \mathrm{O} / \mathrm{H}_{2}}$

$\mathrm{E}_{\mathrm{O}_{2} / \mathrm{OH}^{-}}$

$E_{\text {Redox }}$

$E_{\mathrm{VB}}$

$F$

FF

$G_{i}$

$h$

$I_{0}$

$j_{i}$

$j_{i}^{L}$

$j_{\mathrm{sc}}$

Jell

$J_{0}$

$k_{\mathrm{B}}$

$k_{e^{-}}$

$\mathrm{L}$

$m_{e^{-}}^{*}$ cell area, $\mathrm{m}^{2}$

cell thickness, $\mathrm{m}$

concentration of species $i, \mathrm{~mol} \mathrm{~m}^{-3}$

diffusion coefficient of species $i, \mathrm{~m}^{2} \mathrm{~s}^{-1}$

diffusion coefficient of species $i$ in the bulk electrolyte, $\mathrm{m}^{2} \mathrm{~s}^{-1}$

diffusion coefficient of species $i$ in the electrolyte in the pores of the semiconductor, $\mathrm{m}^{2} \mathrm{~s}^{-1}$

reference diffusion coefficient, $\mathrm{m}^{2} \mathrm{~s}^{-1}$

macroscopic electric field, $\mathrm{V} \mathrm{m}^{-1}$

external bias potential, $\mathrm{V}$

band bending potential, $\mathrm{V}$

conduction band potential, $\mathrm{V}$

flat band potential, $\mathrm{V}$

semiconductor bandgap, $\mathrm{V}$

fermi level of the semiconductor, $V$

water reduction potential, $\mathrm{V}$

water oxidation potential, $\mathrm{V}$

redox potential, $\mathrm{V}$

valence band potential, $\mathrm{V}$

Faraday constant, $9.6485339 \times 10^{4} \mathrm{C} \mathrm{mol}^{-1}$

fill factor

generation rate of species $i, \mathrm{~m}^{-3} \mathrm{~s}^{-1}$

Planck constant, 6.6260693(11) $\times 10^{-34} \mathrm{~J} \mathrm{~s}$

incident photon flux corrected for reflection losses, $\mathrm{m}^{-2} \mathrm{~s}^{-1}$

current density of species $i, \mathrm{~s}^{-1} \mathrm{~m}^{-2}$

current density of species $i$ in the electrolyte in the pores of the semiconductor, $\mathrm{s}^{-1} \mathrm{~m}^{-2}$

short-circuit current density, $\mathrm{A} \mathrm{m}^{-2}$

net current density, $\mathrm{A} \mathrm{m}^{-2}$

exchange current density at Pt electrode, $\mathrm{A} \mathrm{m}^{-2}$

boltzman constant, $1.3806503 \times 10^{-23} \mathrm{~J} \mathrm{~K}^{-1}$

back reaction rate constant, $\mathrm{s}^{-1}$

thickness of the semiconductor, $\mathrm{m}$

effective electron mass, $\mathrm{kg}$ 
$n \quad$ number of electrons transferred in the reaction

$n_{\text {eq }} \quad$ dark equilibrium electron density, $\mathrm{m}^{-3}$

$n_{i} \quad$ density of species $i, \mathrm{~m}^{-3}$

$n_{i}^{b} \quad$ density of species $i$ in the bulk electrolyte, $\mathrm{m}^{-3}$

$n_{i}^{L} \quad$ density of species $i$ in the electrolyte in the pores of the semiconductor, $\mathrm{m}^{-3}$

$n_{\text {int }} \quad$ intrinsic carrier concentration, $\mathrm{m}^{-3}$

$n_{\text {ref }} \quad$ reference particle density, $\mathrm{m}^{-3}$

$N_{C B}$ effective density of states in the conduction band, $\mathrm{m}^{-3}$

$N_{\mathrm{VB}} \quad$ effective density of states in the valence band, $\mathrm{m}^{-3}$

$q$ elementary charge, $1.60217646 \times 10^{-19} \mathrm{C}$

$R_{i} \quad$ recombination rate of species $i, \mathrm{~m}^{-3} \mathrm{~s}^{-1}$

$R_{\text {ohmic }} \quad$ ohmic resistance, $\Omega$

$\mathrm{T}$ absolute temperature, $\mathrm{K}$

$t$ time, s

$\mathrm{V}_{\mathrm{OC}} \quad$ open-circuit voltage, $\mathrm{V}$

$x \quad$ coordinate, $m$

$\alpha(\lambda) \quad$ wavelength-dependent absorption coefficient, $\mathrm{m}^{-1}$

$\beta \quad$ transfer coefficient

$\varepsilon \quad$ dielectric constant

$\varepsilon_{p} \quad$ semiconductor film porosity

$\varepsilon_{0} \quad$ permittivity of free space, $8.85419 \times 10^{-12} \mathrm{~F} \mathrm{~m}^{-1}$

$\eta_{a} \quad$ overpotential at the anode, $\mathrm{V}$

$\eta_{\text {inj }} \quad$ electron injection efficiency

$\eta_{\text {ohmic }} \quad$ ohmic overpotential, V

$\eta_{\mathrm{Pt}} \quad$ overpotential at Pt electrode (cathode), $\mathrm{V}$

$\lambda \quad$ wavelength, $\mathrm{m}$

$\mu_{\mathrm{i}} \quad$ mobility of species $i, \mathrm{~m}^{2} \mathrm{~V}^{-1} \mathrm{~s}^{-1}$

$\mu_{i}^{b} \quad$ mobility of species $i$ in the bulk electrolyte, $\mathrm{m}^{2} \mathrm{~V}^{-1} \mathrm{~s}^{-1}$

$\mu_{i}^{L} \quad$ mobility of species $i$ in the electrolyte in the pores of the semiconductor, $\mathrm{m}^{2} \mathrm{~V}^{-1} \mathrm{~s}^{-1}$

$\nu \quad$ frequency, $\mathrm{s}^{-1}$

$\tau \quad$ tortuosity

$\tau_{n} \quad$ carrier lifetime, $\mathrm{s}$

$\Delta E_{\mathrm{F}} \quad$ variation of the Fermi level potential, $\mathrm{V}$

$\Delta G \quad$ free energy for the overall cell reaction, J

$\theta \quad$ dimensionless time variable

\section{Superscripts}

init initial conditions

$\mathrm{O}^{+} \quad$ point close to the photoanode

$0^{-} \quad$ external point of the current collector

* dimensionless variable

standard

\section{Subscripts \\ $e^{-} \quad$ electrons \\ $h^{+} \quad$ holes}

$\begin{array}{ll}\text { Abbreviations } \\ \text { CB } & \text { conduction band } \\ \text { CE } & \text { counter-electrode } \\ \text { ref } & \text { reference } \\ \text { SC } & \text { semiconductor } \\ \text { PEC } & \text { photoelectrochemical } \\ \text { TCO } & \text { transparent conductor oxide } \\ \text { VB } & \text { valence band }\end{array}$

\section{REFERENCES}

[1] Nathan SL. Basic research needs for solar energy utilization. Renée M. Nault; 2005.

[2] Gratzel M. Nature 2001;414:338.

[3] Krol RVd, Liang Y, Schoonman J. Journal of Materials Chemistry 2008;18:2311.

[4] Trieb F. Concentrating solar power for the Mediterranean region. German Aerospace Center (DLR); 2005.

[5] Nowotny J, Sorrell CC, Sheppard LR, Bak T. International Journal of Hydrogen Energy 2005;30:521.

[6] Cesar I, Kay A, Gonzalez Martinez JA, Grätzel M. Journal of the American Chemical Society 2006;128:4582.

[7] Archer MD, Nozik AJ. Nanostructured and photoelectrochemical systems for solar photon conversion. London/Singapore; Hackensack, NJ: Imperial College Press/ World Scientific Pub. Co.; 2008.

[8] Aroutiounian VM, Arakelyan VM, Shahnazaryan GE, Hovhannisyan HR, Wang H, Turner JA. Solar Energy 2007;81:1369.

[9] Zerta M, Schmidt PR, Stiller C, Landinger H. International Journal of Hydrogen Energy 2008;33:3021.

[10] Aroutiounian VM, Arakelyan VM, Shahnazaryan GE, Stepanyan GM, Turner JA, Khaselev O. International Journal of Hydrogen Energy 2002;27:33.

[11] Nozik AJ. Annual Review of Physical Chemistry 1978;29:189.

[12] Aroutiounian VM, Arakelyan VM, Shahnazaryan GE, Stepanyan GM, Turner JA, Kocha SS. Electrochimica Acta 2000;45:1999.

[13] Boudjemaa A, Boumaza S, Trari M, Bouarab R, Bouguelia A. International Journal of Hydrogen Energy 2009;34:4268.

[14] Glasscock JA, Barnes PRF, Plumb IC, Savvides N. The Journal of Physical Chemistry C 2007;111:16477.

[15] Sivula K, Formal FL, Graìtzel M. Chemistry of Materials 2009; 21:2862.

[16] Gerischer H. Journal of Electroanalytical Chemistry 1975;58:263.

[17] Gerischer H. Journal of Electroanalytical Chemistry 1977;82:133.

[18] Nozik AJ. Applied Physics Letters 1976;29:150.

[19] Khan SUM, Bockris JOM. The Journal of Physical Chemistry 1984;88:2504.

[20] Choi P, Bessarabov DG, Datta R. Solid State Ionics 2004;175: 535.

[21] Nie J, Chen Y, Boehm RF, Katukota S. Journal of Heat Transfer 2008;130:042409.

[22] Marangio F, Santarelli M, Calì M. International Journal of Hydrogen Energy 2009;34:1143.

[23] Ni M. Mathematical modeling of solid oxide steam electrolyzer for hydrogen production. PhD Thesis. University of Hong Kong; 2007.

[24] Ni M, Leung MKH, Leung DYC. International Journal of Hydrogen Energy 2008;33:2337.

[25] Ni M, Leung MKH, Leung DYC. International Journal of Hydrogen Energy 2008;33:4040.

[26] Grimes CA, Varghese OK, Ranjan S. Light, water, hydrogen: the solar generation of hydrogen by water photoelectrolysis. New York: Springer; 2008.

[27] Lindgren T, Vayssieres L, Wang H, Lindquist S-E. In: Kokorin AI, Bahnemann DW, editors. Chemical physics of nanostructured semiconductors. Boston: VSP - An imprint of BRILL; 2003. p. 83.

[28] Archer MD, Nozik AJ. Nanostructured and photoelectrochemical systems for solar photon conversion. London: Imperial College Press; 2008.

[29] Cesar I. Solar photoelectrolysis of water with translucent nano-structured hematite photoanodes. PhD Thesis. EPFL; 2007.

[30] Würfel P. Physics of solar cells: from principles to new concepts. Weinheim; 2005. 
[31] Ferber J, Stangl R, Luther J. Solar Energy Materials and Solar Cells 1998;53:29.

[32] Bard AJ, Faulkner LR. Electrochemical methods: fundamentals and applications. 2nd ed. USA: John Wiley \& Sons, Inc.; 2001.

[33] Baranenko VI, Kirov VS. Atomic Energy 1989;66:30.

[34] Bak T, Nowotny J, Rekas M, Sorrell CC. International Journal of Hydrogen Energy 2002;27:991.

[35] Petzold L. SIAM Journal of Scientific and Statical Computing 1983;4:136.

[36] Cesar I, Sivula K, Kay A, Zboril R, Graetzel M. The Journal of Physical Chemistry C 2009;113:772.

[37] O'Hayre R, Cha S-W, Colella W, Prinz FB. Fuel cells fundamentals. New York: John Wiley \& Sons, Inc.; 2006.

[38] Kay A, Cesar I, Gratzel M. Journal of the American Chemical Society 2006;128:15714.

[39] Bjoerksten U, Moser J, Graetzel M. Chemistry of Materials 1994;6:858.
[40] Liang Y, Enache CS, Krol RVd. International Journal of Photoenergy 2008;2008. doi:10.1155/2008/739864. Article ID 739864, 7 pages.

[41] Dare-Edwards MP, Goodenough JB, Hamnett A, Trevellick PR. Journal of the Chemical Society, Faraday Transactions 1: Physical Chemistry in Condensed Phases 1983;79.

[42] Itoh K, Bockris JOM. Journal of the Electrochemical Society 1984;131:1266.

[43] Cherepy NJ, Liston DB, Lovejoy JA, Deng H, Zhang JZ. The Journal of Physical Chemistry B 1998;102:770.

[44] Bosman AJ, van Daal HJ. Advances in Physics 1970;19:1.

[45] Hamann CH, Hamnett A, Vielstich W. Electrochemistry. 2nd ed. Weinheim: Wiley-VCH Verlag GmbH \& Co. KGaA; 2007.

[46] Larminie J, Dicks A. Fuel cells systems explained. 2nd ed. England: John Wiley \& Sons Ltd; 2003. 